\title{
Can Written Disclosure Reduce Psychological Distress and Increase Objectively Measured Injury Mobility of Student-Athletes? A Randomized Controlled Trial
}

\author{
Elaine Duncan, ${ }^{1}$ Yori Gidron, ${ }^{2}$ and David Lavallee ${ }^{3}$ \\ ${ }^{1}$ Department of Psychology and Allied Health Sciences, Glasgow Caledonian University, Glasgow G4 OBA, UK \\ ${ }^{2}$ Faculty of Medicine \& Pharmacy, Free University of Brussels (VUB), 1050 Brussels, Belgium \\ ${ }^{3}$ School of Sport Stirling, University of Stirling, Stirling FK9 4LA, UK \\ Correspondence should be addressed to David Lavallee; david.lavallee@stir.ac.uk
}

Received 10 May 2013; Accepted 4 June 2013

Academic Editors: K.-H. Lin, M. Schmitter-Edgecombe, and Y. Wu

Copyright (C) 2013 Elaine Duncan et al. This is an open access article distributed under the Creative Commons Attribution License, which permits unrestricted use, distribution, and reproduction in any medium, provided the original work is properly cited.

Injured students-athletes took part in a randomized controlled trial to test whether written disclosure could reduce psychological distress and improve injury mobility. Writing took place alongside prescribed physical rehabilitation and consisted of three 20minute writing sessions, once a week for three consecutive weeks. Participants in the experimental injury-writing group ( $n=25)$ followed a structured form of written disclosure, called the guided disclosure protocol (GDP). They firstly, wrote about the onset of their injury in a chronological manner, secondly, they explicitly labelled their emotions and described the impact of the injury, finally they wrote about future coping and psychological growth. Controls $(n=21)$ wrote about nonemotional and noninjury related topics. In addition to self-report measures, a physiotherapist, blind to experimental condition, assessed mobility at the injury site. Although self-report indices remained unchanged, the GDP group evidenced a significant improvement in injury mobility compared to controls.

\section{Introduction}

Empirical work with athletes reveals that sports injury can cause considerable psychological distress [1-3] and that injured athletes have higher levels of emotional disturbance than noninjured controls [4]. The consequent withdrawal from training and competition can also be destabilizing [5]. Moreover, loss of athletic identity, withdrawal from the social climate of sport, and the possibility that an injury is career ending create an atmosphere of extreme anxiety and isolation which researchers and practitioners liken to a grief process [6-8]. There is also evidence that the emotional milieu of injury impacts upon an athletes' perceived and actual rehabilitation [9]. Moreover, models of injury coping and prevention suggest that poor psychological recovery may leave athletes more susceptible to reinjury $[5,10]$. It is thus widely acknowledged that processing cognitive and emotional distress and helping athletes cope with sport-specific situational factors following injury are essential for adherence to treatment and eventual recovery $[5,11,12]$.
Awareness of such factors has led to an increase in psychological interventions being implemented alongside standard physical rehabilitation protocols. Although, Reese and colleagues applauded such integrated practice they nevertheless queried the efficacy of psychological interventions in sports injury rehabilitation [13]. In a recent review they found that interventions consisting of guided imagery, relaxation, microcounselling skills, acceptance and commitment therapy, and written disclosure reduced various types of injury-related psychological distress. However, Reese and colleagues appealed for more systematic and better designed interventions on the grounds that study density was too low to make useful generalizations and that research was often atheoretical and/or lacked systematic use of randomized controlled trial methodology [13].

Of particular relevance to the present paper were the reviews' findings on written disclosure. For clarity, it is important to note that "written disclosure" may also be known as emotional expression and/or expressive writing. In this type 
of intervention participants are typically asked to write about emotionally upsetting and/or traumatic experiences for fixed time periods. Over 250 studies (including meta-analyses and systematic reviews) have shown that written disclosure interventions, and in particular those that provide guidance on what to write, reduce intrusive thoughts, improve overall mood, and boost immune functioning [14-16]. In addition, improved psychological and physical outcomes have also been found in such clinical populations as cancer patients [17], coronary patients [18], those undergoing a surgical procedure [19], chronic pelvic pain sufferers [20], and those with rheumatoid arthritis [21]. The mechanisms underpinning the effects of written disclosure are still a live debate but consensus that an interaction between biological, social, and psychological mechanisms is most likely $[14,15]$. Given this robust evidence base, it is surprising that only three published studies have utilized written disclosure in a sports context, with only two of these directly related to sports injury rehabilitation. In a qualitative study, Hudson and Day [22] explored athletes' experiences of expressive writing about competitive sport stressors. The study outcomes suggested that expressive writing was a relatively cost efficient and approachable way of helping athletes reframe stressors and manage emotions. Directly related to sports injury rehabilitation are two studies conducted by Mankad and colleagues [23, 24]. In a single case study design written disclosure was used to facilitate coping with severe injury [23]. The participant, a male gridiron footballer, reported improved mood and self-esteem. Narrative analysis highlighted that his writing was suffused with emotion and cognitive understanding of his injury. In a repeated measures design, Mankad et al. [24] asked elite athletes to write about their negative thoughts and feelings surrounding their injury and rehabilitation experience for three consecutive days. Participants reported reduced levels of stress and mood disturbance and improved markers of immunity [24]. Linguistic word count analysis of the athletes' writings revealed fewer grief responses to injury across writing days. Mankad et al. [24] have argued that writing may act as the vehicle for the emotional and cognitive processing necessary for psychological adjustment to and recovery from injury [13, 25]. However, Mankad [26] recommended further experimental trials and in particular those that included objective measurement of injury status alongside assessment of psychological outcomes.

In sum, preliminary research suggests that written disclosure is associated with positive outcomes in a sports injury context and that the time is right for expansion of research in this area [22]. The present study hoped to add to research investigating the efficacy of psychological interventions in sports injury rehabilitation with particular focus on whether a guided instructions form of written disclosure could reduce psychological distress and improve objectively measured injury mobility. Evidence suggests that the potential benefits of written disclosure can be optimized when participants are offered guidance on what to write, $[14,27]$ so our participants adhered to a guided disclosure protocol (GDP) designed by Duncan and Gidron [28].

The GDP attempts to embody some of the positive findings from research on written disclosure. In particular that participants should receive specific instructions that encourage them to write an organized self-reflective narrative and that writing is best conducted under timed and controlled conditions. The design and rationale of the GDP is also rooted in the theoretical and empirical work on the dynamics of traumatic memory encoding. Specifically, the contention that shifting such memories from implicit and limbic to explicit and more cortical recall may be beneficial via cognitive processing of affective and somatic experiences [32, 33]. Finally, the GDP instructions incorporate the principles of reappraisal, benefit finding, and self-regulation. Specifically, adopting multiple perspectives of an event and reviewing its capacity to provide adaptive schemas and resources to deal with similar future events are useful [34-36].

The GDP has already been found to reduce clinic visits in frequent clinic attendees [37] and reduce psychological distress in parents of children with cancer [38]. We propose that the GDP offers a framework for the processing and alleviation of the emotional and cognitive consequences associated with injury in a sports context. Evidence, cited above, suggests that writing can also improve various physical parameters. Hence, the present study assessed mobility status of the presenting injuries. It was hypothesized that the GDP, injury-writing participants would report reduced levels of psychological distress and enhanced mobility in comparison to those writing about nonemotive and noninjury related topics.

To increase methodological rigour, the study was a randomized controlled trial. In addition to self-report indices of psychological distress, a physiotherapist, blind to experimental condition, objectively measured mobility at the injury site. Given the infancy of research on writing and sports injury, it was felt more appropriate to include a wide variety of injuries, as opposed to screening for one injury type only. The sample was injured student-athletes.

1.1. Participants and Recruitment. Participants were recruited from the student population attending a large university in the United Kingdom. Informed consent and local ethics committee procedures and practice were followed and established in full for all participants. Recruitment was achieved via the placement of leaflets within the university's rehabilitation facilities. These leaflets contained a brief outline of the study, the extent of participant commitment, and inclusion criteria. To be included participants had to be 18 years of age or over, to have been injured during competition and/or training, to have an injury that forced cessation of training and competition, and be attending physical rehabilitation. Participants were trusted to self-assess whether they fulfilled such inclusion criteria and to make contact with the researcher. One further inclusion criteria was that our participants should be those whose self-worth was highly invested in their sport. Previous research and theory suggest that athletes who define themselves primarily through their sport are likely to experience higher levels of distress than those whose perception of self-worth is not exclusively drawn from identification with the athletic role $[39,40]$. Thus, only those with a strong and exclusive identification with the athlete role (i.e., by scoring above 30 on the athletic identity measurement scale; see 
description of scales below) were invited to continue into the writing intervention itself. Those not meeting this threshold continued with physical rehabilitation as normal but were excluded from the writing intervention. We made no attempt to select for particular sports.

\section{Method}

Forty-six participants were randomly assigned to the nonemotive, noninjury writing control group $(n=21)$ or the GDP, injury-writing group $(n=25)$. Participants completed preintervention (baseline) and postintervention outcome measures of psychological distress. The study physiotherapist, blind to experimental condition, measured mobility at five time points: prior to the writing (baseline), after each of the three writing sessions, (writing days, 1, 2, and 3) and after writing (postintervention). The study recruitment period spanned six months. The time lapse between injury onset, the start of physical rehabilitation and involvement in the writing intervention was two weeks.

2.1. Procedure. All consenting participants were invited to complete the athletic identity measurement scale (AIMS) [39]. Those scoring above 30 and giving consent to go forward into the intervention were randomly assigned to the GDP, injury-writing group or the nonemotive, noninjury writing control group. Those not reaching this threshold were debriefed, thanked for their time, and continued with their prescribed physical rehabilitation as normal.

In order to complete the various indices of emotional distress, writing participants met with a researcher on five separate occasions. These were timed to coincide with their scheduled physical treatment. On each occasion, participants were given oral and written instructions. These differed per group and for each of the three sessions (see intervention instructions below). For both groups, writing sessions took place once a week for three consecutive weeks on the day of and immediately prior to their prescribed physiotherapy treatment. Participants wrote at a desk in a private room adjacent to where physiotherapy took place. Each writing session lasted 20 minutes. All writing sessions were supervised and timed by a researcher and conducted on an individual, not group, basis. Fresh writing materials were provided at each writing session. Participants did not have access to their previous writings. The researcher collected the writings at the end of each session. Participants were assigned a unique participant number and advised to keep writing free of personal identifiers. Participants were reassured that the content of their stories would be kept confidential and viewed only by the research team should content analysis of actual writings be deemed appropriate at a later date.

All those taking part in the writing intervention itself completed a battery of self-report indices at preintervention (baseline), on each of the three writing days and at a postintervention stage. The time lapse between completion of outcome measures at baseline, the start and/or end of the intervention was no more than two weeks. At the postintervention stage, participants were also asked how valuable/meaningful the writing sessions were, how much they had talked to others about what they had written, and how much they had thought about what they had written. These were each rated on a 7-point Likert scale where, for example, 1 = very valuable/meaningful and where $7=$ not at all valuable/meaningful. Experiential questions of this nature are a norm in written-disclosure studies, providing feedback on the experience of writing itself.

The self-report indices were the Reinjury Anxiety Inventory (RIAI) [41, 42]; 30-item mood questionnaires [43], and the centre for Epidemiological Studies on Depression Scale (CESD-10) [44]. In addition to pre and postintervention mood-sampling, participants completed the same mood questionnaire immediately following each of the three 20minute writing sessions. This provided a measure of mood at five points: prior to the writing intervention, (baseline) immediately following each of the three writing sessions (writing days, 1, 2, and 3), and approximately two weeks after writing (postintervention).

Mobility at the injury site was measured at these same five time points. During the three-week writing phase, measurement was taken immediately after the timed writing sessions. When measuring mobility, the physiotherapist adhered to standard protocols, such as ensuring each participant had adequate time to warm up and cool down. No changes were made to the physiotherapists' prescribed treatment and/or their way of working with a client.

\subsection{Intervention Instructions}

2.2.1. Instructions to Participants in the GDP, Injury-Writing Group. As described in the procedure above, participants wrote on three separate occasions at weekly intervals. The sessions lasted 20 minutes. An outline of the instructions is provided, but for verbatim instructions please contact the first author. On the first day of writing (writing day 1) GDP participants wrote in a chronological and nonemotive manner about the occurrence of the injury. They were asked to provide an account of when the injury happened, the circumstances around its occurrence and to do so in a detached, objectively descriptive way. On writing day 2 (one week later), participants were asked to write about the emotions and feelings at the time of injury and its immediate impact on day-to-day life. Finally, in the third week of the intervention (writing day 3), participants were guided to write about their current perspective on the injury, possible psychological growth, and future coping.

\subsubsection{Instructions to Participants in the Nonemotive, Nonin-} jury Writing Group. Participants in the control group were also asked to engage in writing on three separate occasions spaced across a three-week period. Participants in this group were guided to write about nonemotive, noninjury related topics. Thus, on writing day 1 , they were invited to write about what they had done since waking up that morning, on the second, to detail a recent social event, and on the third, their plans for the remainder of the day. On each occasion, it was stressed that they describe things exactly as they occurred or 
would occur, not to mention emotions, feelings, or opinions, but to be as objective as possible. Verbatim instructions for the control group are available by contacting the first author.

\subsection{Description of Measures}

2.3.1. Injury Mobility. We examined mobility across a range of injuries experienced by study participants. Mobility at the injury site was measured on five occasions with a Cybex 330 isokinetic exercise and monitoring machine. Isokinetic concentric peak torque measurements (in Newton-meters) were taken prior to the intervention, after each of the three writing sessions, and after the intervention.

2.3.2. Adherence to Rehabilitation. The physiotherapist also recorded the level of adherence to rehabilitation of each participant. This was simply recorded as the number of sessions actually attended, expressed as a percentage of total visits prescribed.

\subsubsection{Psychological Measures}

Athletic Identity Measurement Scale. This scale measures the strength and exclusivity of identification with the athletic role [39]. It is a 10-item scale with ratings on a 7-point Likert scale. The scale includes elements of athletic identity such as social (e.g., "Most of my friends are athletes"), cognitive (e.g., "I have many goals related to sport"), and affective (e.g., "I feel bad about myself when I do poorly in sport"). Final ratings were summed to provide an overall athletic identity score. The psychometric integrity of the AIMS has been provided through previous research [39]. This scale was used as part of the inclusion criteria as described above.

Reinjury Anxiety Inventory [41]. This scale probes the degree to which already injured athletes fear reinjury and has been shown to be a reliable and valid measure [42]. The inventory contains 28 items (on a $0-3$ Likert scale, where $0=$ not at all and 3 = very much so) and two subscales: reinjury anxiety in rehabilitation (RIA-R) and reinjury anxiety regarding reentry into competition (RIA-RE). The RIA-R has a range of $0-39$, whereas the RIA-RE has a range of $0-45$ with lower scores indicating less anxiety on both subscales.

Mood Questionnaire [43]. Positive and negative mood was measured at baseline and postintervention and following each writing session. This scale is a 30 -item scale with equal numbers of positive and negative mood dimensions. Scoring is accomplished by summing points on a 5-point Likert scale for the 15 items pertaining to each mood dimension.

Centre of Epidemiological Studies Depression Scale [44]. This is designed to measure depressive symptomology in the general population. The original scale had 20 items, but the 10 -item scale was chosen to lessen the burden on participants and because reliability statistics indicate that shorter versions of the scale do not sacrifice precision [45].

The decision to include indices of general mood, depressive symptomology, and fears around injury was not an arbitrary one, but informed by previous research into the experience and consequences of sports injury, cited above. For the purposes of this study these scales constituted our measure of psychological distress.

\section{Results}

3.1. Descriptive Statistics and Baseline Group Equality. Table 1(a) depicts the general categorical characteristics for the entire sample. Table 1(b) depicts the entire sample's data on continuous measures. The table lists the type of injuries presented in rehabilitation. Knee injury was the most common, followed by injuries sustained to the ankle area. Participants also presented with injuries to the shoulder, lower leg, and back.

Table 2 depicts the baseline and postintervention scores on the study outcomes, per group. At baseline, the GDP injury writing group's score on AIMS was significantly higher than that of controls $(t(44)=2.48, P<.05)$. Similarly, this group RAI-RE score was significantly higher than that of controls $(t(42)=2.48, P<.05)$. Thus, in the main outcome analyses, AIMS and RAI-RE were statistically controlled for. No other continuous background or outcome variable differed significantly between groups at baseline. Groups did not differ on gender or adherence to rehabilitation.

3.2. Main Outcome Analyses. Table 3 depicts the results of the mixed-design analyses of covariance (ANCOVA), controlling for baseline AIMS, and RAI-RE. A statistically significant time $\times$ group interaction was found for mobility, even when controlling for baseline mobility. Simple-effects analyses revealed that groups differed significantly on mobility at writing day $1(F(1,32)=10.8, P<.005)$; at writing day $2(F(1,31)=$ $17.6, P<.001)$, at writing day $3(F(1,33)=22.2, P<.001)$, and at the postintervention measurement $(F(1,32)=26.2$, $P<.001)$. This interaction is depicted in Figure 1. In Figure 1, numbers $1,2,3$, and 4 represent writing day 1 through to the postintervention measurement. Table 3 also depicts that time tended to interact with group in relation to depressive symptoms $(P=.06)$ but this change was not statistically significant.

3.3. Post-Writing Evaluation. Participants in the GDP group reported significantly higher scores to the questions on how meaningful and how valuable the writing had been when compared to the controls. Though not statistically significant, GDP participants tended to think more about their writing than controls $(t(42)=1.77, P<.10)$ but did not differ on talking about the written content with others from controls $(t(42)=1.04)$.

\section{Discussion}

Evidence suggested that various forms of psychological interventions, including written disclosure could be useful adjuncts to standard physical rehabilitation in a sports context [13]. However, despite a strong evidence base only two 
TABLE 1: Sample characteristics.

(a) Categorical data

\begin{tabular}{lc}
\hline Variable & $\%$ \\
\hline Gender & \\
Men & 58.7 \\
Women & 41.3 \\
Locus of injury & \\
Knee & 48.8 \\
Ankle & 30.2 \\
Lower leg & 11.6 \\
Shoulder & 7.0 \\
Back & 2.3 \\
Adherence & \\
None & 6.5 \\
$60 \%$ & 6.5 \\
$80 \%$ & 21.7 \\
$100 \%$ & 65.2 \\
\hline
\end{tabular}

(b) Continuous data (baseline)

\begin{tabular}{lcc}
\hline Variable & Mean & SD \\
\hline Age & 21.5 & 2.9 \\
AIMS & 31.2 & 5.6 \\
Mood-a & 27.1 & 4.3 \\
Mood-b & 27.3 & 4.4 \\
Depressive symptoms & 20.8 & 3.7 \\
RAI-R & 15.5 & 8.1 \\
RAI-RE & 22.5 & 9.1 \\
Mobility & 30.1 & 14.1 \\
\hline
\end{tabular}

Note. AIMS: athletic identity measurement scale; Mood-a: positive emotion; Mood-b: negative emotion; RAI-R: reinjury anxiety in rehabilitation; RAI-RE: reinjury anxiety reentry into competition.

studies to date had sought to use writing in a sports injury rehabilitation context $[23,24]$. Aiming to extend research, the present study investigated whether a structured form of writing (GDP) could decrease psychological distress and increase injury mobility in student-athletes. Although, none of the self-report measures were differently affected by groups' mobility those in the GDP group improved almost three times compared to controls. These effects were independent of baseline AIMS, RAI-RE, and baseline mobility.

We believe that this is only the third study to have implemented written disclosure in a sports injury rehabilitation context. Although, the present study and those carried out by Mankad and colleagues found positive results major differences between the studies make direct comparison difficult. The sample in the present study was drawn from a population of student-athletes whereas those in Mankad's studies were described as elite level athletes. Although, each study contained measures of psychological and physical indices the types of instruments and procedures used varied as did the writing instructions. Unlike the others, the present study operationalized writing within randomized controlled trial methodology and included an objectively measured outcome. Despite such differences each study and the present
TABLE 2: Baseline background information and pre- and postintervention results on outcomes per study group.

\begin{tabular}{lcccc}
\hline \multirow{2}{*}{ Variable } & \multicolumn{2}{c}{ GDP } & \multicolumn{2}{c}{ Control } \\
& Mean & SD & Mean & SD \\
\hline Age & 21.2 & 3.0 & 21.9 & 2.9 \\
Adherence & 87.2 & 22.3 & 85.7 & 30.4 \\
AIMS & 39.0 & 5.3 & 35.0 & $5.3^{*}$ \\
Mood-a-baseline/preintervention & 27.8 & 3.6 & 26.3 & 4.9 \\
Mood-a-writing day 1 & 23.5 & 4.5 & 21.2 & 5.8 \\
Mood-a-writing day 2 & 24.3 & 4.0 & 22.5 & 5.9 \\
Mood-a-writing day 3 & 23.3 & 4.2 & 21.8 & 5.9 \\
Mood-a-time 4/postintervention & 26.8 & 5.0 & 28.4 & 6.1 \\
Mood-b-baseline/preintervention & 28.1 & 4.1 & 26.5 & 4.5 \\
Mood-b-writing day 1 & 24.0 & 4.2 & 23.2 & 5.4 \\
Mood-b-writing day 2 & 25.7 & 4.8 & 23.4 & 4.7 \\
Mood-b-writing day 3 & 24.7 & 4.9 & 23.4 & 5.2 \\
Mood-b-time 4/postintervention & 26.0 & 5.9 & 25.9 & 5.4 \\
Depression-baseline & 9.2 & 4.5 & 8.8 & 4.7 \\
Depression-post & 9.3 & 5.8 & 7.7 & 3.7 \\
RAI-R-baseline & 16.8 & 7.5 & 13.7 & 8.7 \\
RAI-R-post & 16.9 & 7.6 & 13.2 & 9.8 \\
RAI-RE-baseline & 25.4 & 8.4 & 18.8 & $9.0 *$ \\
RAI-RE-post & 24.5 & 8.8 & 18.0 & 8.6 \\
Mobility-baseline/preintervention & 27.7 & 11.8 & 33.3 & 16.4 \\
Mobility-writing day 1 & 3.8 & 2.5 & 1.8 & 1.4 \\
Mobility-writing day 2 & 5.4 & 2.1 & 2.5 & 1.3 \\
Mobility-writing day 3 & 8.4 & 3.3 & 3.2 & 1.5 \\
Mobility-time 4/postintervention & 9.7 & 4.4 & 3.6 & 1.5 \\
\hline Note: <.05; P <.0. & & & &
\end{tabular}

Note: ${ }^{*} P<.05 ;{ }^{* *} P<.01$.

GDP: Guided Disclosure Protocol. AIMS: athletic identity measurement scale; RAI-R: reinjury anxiety in rehabilitation; RAI-RE: reinjury anxiety reentry into competition; Mood-a: positive emotion; Mood-b: negative emotion.

TABLE 3: Results of analyses of covariance testing interaction effects of group (GDP, control) by time since writing on study outcomes, controlling for covariates.

\begin{tabular}{lcccc}
\hline Outcome & Effect & $F$ & DF & Significance \\
\hline Mobility & Time $\times$ Group & 16.36 & 3,69 & .000 \\
Depression & Time $\times$ Group & 3.73 & 1,36 & .06 \\
\hline
\end{tabular}

provide evidence of gains from writing and how easy it is to implement such interventions.

Despite evidence that writing improves psychological outcomes in many diverse clinical and nonclinical samples, [14] our judgment of its ability to do so within a sports injury rehabilitation context would need to be a conservative one. More research is needed before declaring that written disclosure can be used alongside physical rehabilitation protocols to ameliorate psychological distress. Yet this study informed us that it possible to engage injured student athletes in the process of working through injury related emotions and cognitions via structured written disclosure.

It is also worth noting that evidence of positive change in physical but not psychological outcomes is not an unfamiliar 


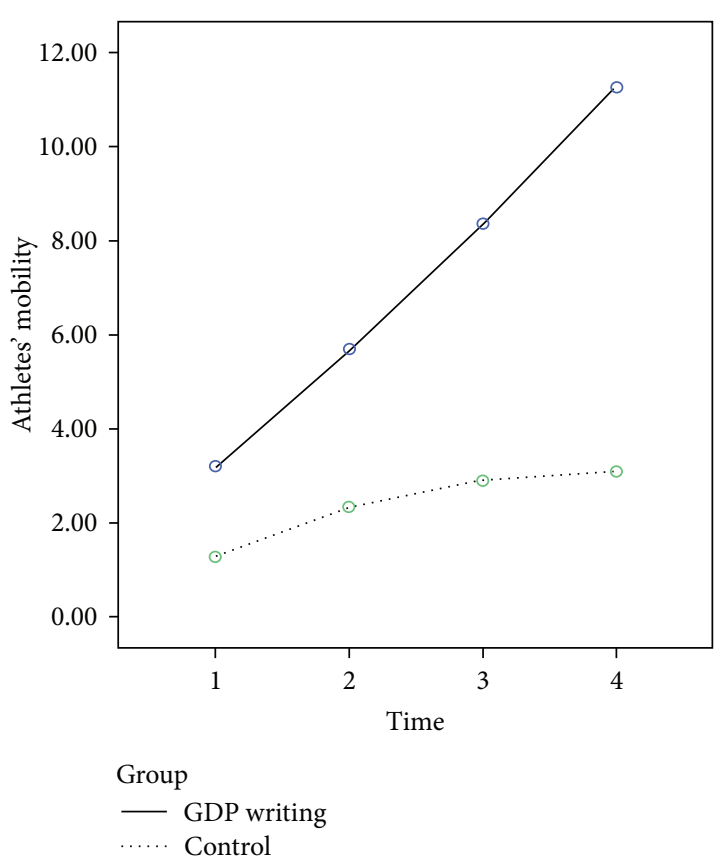

FIGURE 1: Effects of group and time on athletes' mobility.

pattern in writing studies $[17,21]$. For example, Weinman and colleagues [46] investigated whether written disclosure could speed the progress of wound healing in healthy participants. All participants had agreed to have a $4 \mathrm{~mm}$ skin biopsy taken in the upper area of their nondominant arm. Participants in the emotional disclosure group evidenced significantly smaller wounds 14 and 21 days following the biopsy compared to participants who wrote about time management. Despite significant improvement in the main physical outcome, emotional disclosure had no effect on levels of the study's selfreport outcomes, perceived stress, emotional distress, and health behaviours. Moreover, an authoritative review of 146 written disclosure studies noted that written disclosure does not always evidence changes in perceived stress or health behaviour even in the face of physical improvement [14]. Of course it is possible that the psychological inventories we used were not sensitive enough to capture any change in the psychological responses to injury in this particular sample. Yet our psychological measures were not atypical to those of previous sports injury studies where improved psychological outcomes have occurred [13]. It may also be the case that changes in such psychological indices would only be apparent in those who experienced much higher levels of injuryrelated distress than participants in our study.

It is the case that variation in such factors as dosage, the writing instructions, and the setting in which writing takes place moderate the outcomes of writing interventions [14]. It is therefore possible that our findings are an artefact of differences in the design and implementation of our intervention. For example, rather than three consecutive days of writing our participants adhered to a once a week for three weeks dosage. In addition our GDP instructions form part of a more structured form of disclosure than is typically seen in writing studies. In guiding our participants to write about day-to-day impact and possible psychological growth we encourage them to move beyond mere emotional catharsis and this may have implications for mood and other psychological processes. These factors may have contributed to the differential pattern of our outcomes but until we conduct a study with sufficient power and experimental conditions that address these factors we have no evidence that they moderated our main outcomes.

Nevertheless, the absence of effects on psychological outcomes makes accounting for the mechanisms underlying the improved mobility of our participants challenging. It is possible to speculate that mobility improvement was driven by upregulation of biological mediators. Indeed, one of the mechanisms by which expressive writing is said to work is that it lessens overall physiological stress, thereby promoting better physical functioning (15). We know that studies have shown that higher levels of psychological distress are related to slower wound healing [47]. This may occur due to the dysregulation of various derivatives of immunity such as insufficient interleukin-1 (IL-1) or matrix metalloproteinase [48], which are pivotal in wound healing [49]. Although psychological stress is related to inflammatory and molecular variables which influence healing [50], it is possible that the psychological tests used in the present study did not map onto such local molecular changes. However, the present study did not include measures of such biological mediators, consequently the proposal that writing aided immunity and inflammatory processes can be no more than speculation. In addition, we did not account for other factors that may impact on immunity and thereby tissue repair, such as health behaviours and sleep quality.

Although attendance at rehabilitation reached ceiling for both groups, it is possible that the GDP group adhered better to treatment protocols between sessions, but this was not assessed. Future studies may wish to track athletes adherence to treatment protocols between rehabilitation visits to test whether it may account for the changes in mobility observed in the present study. Nevertheless, our results add support to previous research on the positive association between rehabilitation adherence and rehabilitation outcome [51].

We took no account of preinjury psychological profile and previous history of injury and we do not know when, or if, these student-athletes returned to training and competition. Nevertheless, we observed significant improvements in mobility, using an independent rater, blind to group status, in a randomized controlled trial. In addition postintervention feedback informed us that writing about the thoughts and feelings surrounding injury was valuable and meaningful. The present study may have been enhanced by the inclusion of content analysis of the GDP participants' writing to check for linguistic markers of change in emotion and cognitions in relation to their injury.

\section{Conclusion}

Research into the benefits of written disclosure in a sportsinjury context is in its infancy. The present study is the first 
to implement randomized controlled trial methodology in order to test the efficacy of written disclosure and the first to use objective measurement of mobility alongside writing. The findings of the present study, though positive with respect to mobility, did not provide direct evidence that the GDP alleviates self-reported psychological distress. Results showed significant change in injury status but the exact mechanisms of such changes are unclear. This study demonstrated that it is possible to engage injured athletes in a short-term, writing intervention in the early stages of prescribed physical rehabilitation. However, future writing studies that follow athletes to the end of rehabilitation and into the return to competition phase are needed. Including measures of immunity may provide more direct evidence of underlying mechanisms than are presently available. Finally, the GDP is not the only form of written disclosure thus future studies could compare its efficacy against other types of writing protocols within a sports injury and rehabilitation context.

\section{References}

[1] J. Tracey, "The emotional response to the injury and rehabilitation process," Journal of Applied Sport Psychology, vol. 15, no. 4, pp. 279-293, 2003.

[2] F. Carson and R. Polman, "Experiences of professional rugby union players returning to competition following anterior cruciate ligament reconstruction," Physical Therapy in Sport, vol. 13, no. 1, pp. 35-40, 2012.

[3] R. N. Appaneal, B. R. Levine, F. M. Perna, and J. L. Roh, "Measuring postinjury depression among male and female competitive athletes," Journal of Sport and Exercise Psychology, vol. 31, no. 1, pp. 60-76, 2009.

[4] B. W. Brewer, "Psychology of sport injury rehabilitation," in Handbook of Sport Psychology, R. N. Singer, H. A. Hausenblas, and C. M. Janelle, Eds., John Wiley \& Sons, New York, NY, USA, 2001.

[5] M. Arvinen-Barrow and N. Walker, Eds., The Psychology of Sport Injury and Rehabilitation, Routledge, London, UK, 2012.

[6] L. Evans and L. Hardy, "Sport injury and grief responses: a review," Journal of Sport and Exercise Psychology, vol. 17, no. 3, pp. 227-245, 1995.

[7] P. Edouard, J. Pruvost, J. Edouard, and J. Morin, "Causes of dropouts in decathlon. A pilot study," Physical Therapy in Sport, vol. 11, no. 4, pp. 133-135, 2010.

[8] A. C. Cartoni, C. Minganti, and A. Zelli, "Gender, age and professional-level differences in the psychological correlates of fear of injury in Italian gymnasts," Journal of Sport Behaviour, vol. 28, no. 1, pp. 3-17, 2005.

[9] M. A. Morrey, M. J. Stuart, A. M. Smith, and D. M. WieseBjornstal, "A longitudinal examination of athletes' emotional and cognitive responses to anterior cruciate ligament injury," Clinical Journal of Sport Medicine, vol. 9, no. 2, pp. 63-69, 1999.

[10] D. M. Wiese-Bjornstal, "Psychology and socioculture affect injury risk, response, and recovery in high-intensity athletes: a consensus statement," Scandinavian Journal of Medicine and Science in Sports, vol. 20, supplement 2, pp. 103-111, 2010.

[11] D. M. Wiese-Bjornstal, A. M. Smith, S. M. Shaffer, and M. A. Morrey, "An integrated model of response to sport injury: psychological and sociological dynamics," Journal of Applied Scientific Medicine, vol. 10, no. 1, pp. 46-69, 1998.
[12] M. S. Hagger, M. Griffin, N. L. D. Chatzisarantis, and J. Thatcher, "Injury representations, coping, emotions, and functional outcomes in athletes with sports-related injuries: a test of selfregulation theory," Journal of Applied Social Psychology, vol. 35, no. 11, pp. 2345-2374, 2005.

[13] L. M. S. Reese, R. Pittsinger, and J. Yang, "Effectiveness of psychological interventions following sport injury," Journal of Sport and Health Science, vol. 1, no. 2, pp. 71-70, 2012.

[14] J. Frattaroli, "Experimental disclosure and its moderators: a meta-analysis," Psychological Bulletin, vol. 132, no. 6, pp. 823$865,2006$.

[15] K. A. Baikie and K. Wilhelm, "Emotional and physical health benefits of expressive writing," Advances in Psychiatric Treatment, vol. 11, no. 5, pp. 338-346, 2005.

[16] J. E. Owen, E. R. Hanson, D. A. Preddy, and E. O. Bantum, "Linguistically-tailored video feedback increases total and positive emotional expression in a structured writing task," Computers in Human Behavior, vol. 27, no. 2, pp. 874-882, 2011.

[17] C. E. Mosher, K. N. DuHamel, J. Lam et al., "Randomised trial of expressive writing for distressed metastatic breast cancer patients," Psychology and Health, vol. 27, no. 1, pp. 88-100, 2012.

[18] G. M. Manzoni, G. Castelnuovo, and E. Molinari, "The WRITTEN-HEART study (expressive writing for heart healing): rationale and design of a randomized controlled clinical trial of expressive writing in coronary patients referred to residential cardiac rehabilitation," Health and Quality of Life Outcomes, vol. 9, article 51, 2011.

[19] L. Solano, V. Donati, F. Pecci, S. Persichetti, and A. Colaci, "Postoperative course after papilloma resection: effects of written disclosure of the experience in subjects with different alexithymia levels," Psychosomatic Medicine, vol. 65, no. 3, pp. 477-484, 2003.

[20] S. A. Norman, M. A. Lumley, J. A. Dooley, and M. P. Diamond, "For whom does it work? Moderators of the effects of written emotional disclosure in a randomized trial among women with chronic pelvic pain," Psychosomatic Medicine, vol. 66, no. 2, pp. 174-183, 2004.

[21] J. E. Kelley, M. A. Lumley, and J. C. C. Leisen, "Health effects of emotional disclosure in rheumatoid arthritis patients," Health Psychology, vol. 16, no. 4, pp. 331-340, 1997.

[22] J. Hudson and M. C. Day, "Athletes' experiences of expressive writing about sports stressors," Psychology of Sport and Exercise, vol. 13, no. 6, pp. 798-806, 2012.

[23] A. Mankad and S. Gordon, "Psycholinguistic changes in athletes' grief response to injury after written emotional disclosure," Journal of Sport Rehabilitation, vol. 19, no. 3, pp. 328-342, 2010.

[24] A. Mankad, S. Gordon, and K. E. Wallman, "Psycholinguistic analysis of emotional disclosure: a case study in sport injury," Journal of Clinical Sports Psychology, vol. 3, no. 2, pp. 182-196, 2009.

[25] D. Durso-Cupal, "Psychological interventions in sport injury prevention and rehabilitation," Journal of Applied Sport Psychology, vol. 10, no. 1, pp. 103-123, 1998.

[26] A. Mankad, The role of inhibition and written disclosure in sport injury rehabilitation [Unpublished thesis], 2009.

[27] N. Vrielynck, P. Philippot, and B. Rimé, "Level of processing modulates benefits of writing about stressful events: comparing generic and specific recall," Cognition and Emotion, vol. 24, no. 7, pp. 1117-1132, 2010.

[28] E. Duncan and Y. Gidron, "Written emotional expression and health: evidence for a new guided-disclosure technique," 
Proceedings of the British Psychological Society, vol. 7, no. 1, p. 29, 1999.

[29] C. de Moor, J. Sterner, M. Hall et al., "A pilot study of the effects of expressive writing on psychological and behavioral adjustment in patients enrolled in a phase II trial of vaccine therapy for metastatic renal cell carcinoma," Health Psychology, vol. 21, no. 6, pp. 615-619, 2002.

[30] J. W. Pennebaker and J. R. Susman, "Disclosure of traumas and psychosomatic processes," Social Science and Medicine, vol. 26, no. 3, pp. 327-332, 1988.

[31] M. J. A. Schoutrop, A. Lange, G. Hanewald, U. Davidovich, and H. Salomon, "Structured writing and processing major stressful events: a controlled trial," Psychotherapy and Psychosomatics, vol. 71, no. 3, pp. 151-157, 2002.

[32] E. B. Foa, C. Molnar, and L. Cashman, "Change in rape narratives during exposure therapy for posttraumatic stress disorder," Journal of Traumatic Stress, vol. 8, no. 4, pp. 675-690, 1995.

[33] A. R. Moradi, M. R. Taghavi, H. T. N. Doost, W. Yule, and T. Dalgleish, "Performance of children and adolescents with PTSD on the Stroop colour-naming task," Psychological Medicine, vol. 29, no. 2, pp. 415-419, 1999.

[34] J. E. Bower, M. E. Kemeny, S. E. Taylor, and J. L. Fahey, "Finding positive meaning and its association with natural killer cell cytotoxicity among participants in a bereavement-related disclosure intervention," Annals of Behavioral Medicine, vol. 25, no. 2, pp. 146-155, 2003.

[35] M. A. Greenberg, C. B. Wortman, and A. A. Stone, "Emotional expression and physical health: revising traumatic memories or fostering self-regulation?" Journal of Personality and Social Psychology, vol. 71, no. 3, pp. 588-602, 1996.

[36] L. J. Warner, M. A. Lumley, R. J. Casey et al., "Health effects of written emotional disclosure in adolescents with asthma: a randomized, controlled trial," Journal of Pediatric Psychology, vol. 31, no. 6, pp. 557-568, 2006.

[37] Y. Gidron, E. Duncan, A. Lazar, A. Biderman, H. Tandeter, and P. Shvartzman, "Effects of guided written disclosure of stressful experiences on clinic visits and symptoms in frequent clinic attenders," Family Practice, vol. 19, no. 2, pp. 161-166, 2002.

[38] E. Duncan, Y. Gidron, E. Rabin, L. Gouchberg, A. M. Moser, and J. Kapelushnik, "The effects of guided written disclosure on psychological symptoms among parents of children with cancer," Journal of Family Nursing, vol. 13, no. 3, pp. 370-384, 2007.

[39] B. W. Brewer, J. L. van Raalte, and D. W. Linder, "Athletic identity: Hercules' muscles or Achilles' heel?" International Journal of Sport Psychology, vol. 24, pp. 237-254, 1993.

[40] P. D. Howe, Sport, Professionalism and Pain: Ethnographies of Injury and Risk, Routledge, London, UK, 2004.

[41] N. Walker, J. Thatcher, and D. Lavallee, "A preliminary development of the re-injury anxiety inventory (RIAI)," Physical Therapy in Sport, vol. 11, no. 1, pp. 23-29, 2010.

[42] N. Walker and J. Thatcher, "The emotional response to athletic injury: re-injury anxiety," in Coping and Emotion in Sport, J. Thatcher, D. Lavallee, and M. Jones, Eds., pp. 236-260, Routledge, Oxford, UK, 2012.

[43] M. A. Zevon and A. Tellegen, "The structure of mood change: an idiographic/nomothetic analysis," Journal of Personality and Social Psychology, vol. 43, no. 1, pp. 111-122, 1982.

[44] L. S. Radloff, “The CES-D scale: a self-report depression scale for research in the general population," Applied Psychological Measurement, vol. 1, pp. 385-401, 1977.
[45] E. M. Andresen, J. A. Malmgren, W. B. Carter, and D. L. Patrick, "Screening for depression in well older adults: evaluation of a short form of the CES-D," The American Journal of Preventive Medicine, vol. 10, no. 2, pp. 77-84, 1994.

[46] J. Weinman, M. Ebrecht, S. Scott, J. Walburn, and M. Dyson, "Enhanced wound healing after emotional disclosure intervention," The British Journal of Health Psychology, vol. 13, no. 1, pp. 95-102, 2008.

[47] L. V. Doering, D. K. Moser, W. Lemankiewicz, C. Luper, and S. Khan, "Depression, healing, and recovery from coronary artery bypass surgery," The American Journal of Critical Care, vol. 14, no. 4, pp. 316-324, 2005.

[48] Y. Y. Yang, H. F. Tsai, S. C. Lu, Y. F. Huang, and Y. C. Chang, "Regulation of tissue inhibitors of metalloproteinase-1 gene expression by cytokines in human gingival fibroblasts," Journal of Endodontics, vol. 28, no. 12, pp. 803-805, 2002.

[49] P. T. Marucha, J. K. Kiecolt-Glaser, and M. Favagehi, "Mucosal wound healing is impaired by examination stress," Psychosomatic Medicine, vol. 60, no. 3, pp. 362-365, 1998.

[50] M. Maes, C. Song, A. Lin et al., “The effects of psychological stress on humans: increased production of pro-inflammatory cytokines and a Thl-like response in stress-induced anxiety," Cytokine, vol. 10, no. 4, pp. 313-318, 1998.

[51] M. J. Satterfield, D. Dowden, and K. Yasumura, "Patient compliance for successful stress fracture rehabilitation," Journal of Orthopaedic and Sports Physical Therapy, vol. 11, no. 7, pp. 321325, 1990. 


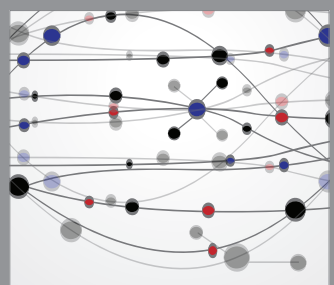

The Scientific World Journal
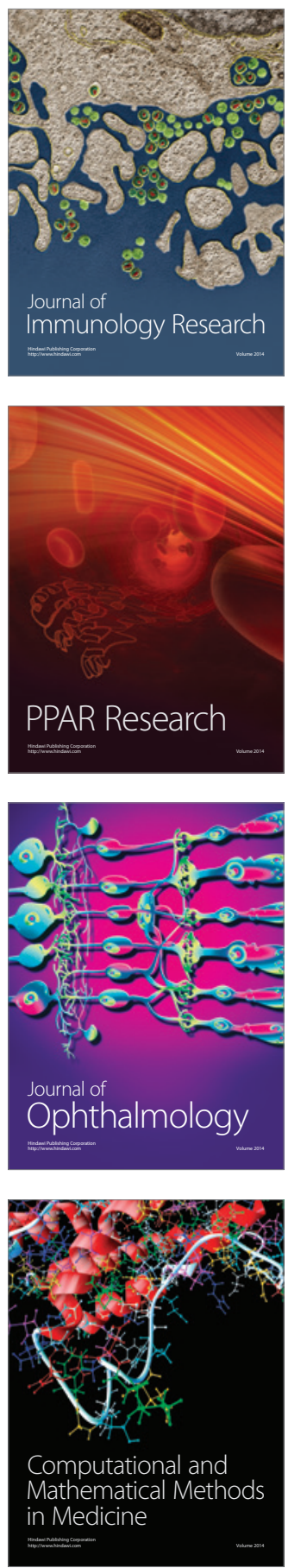

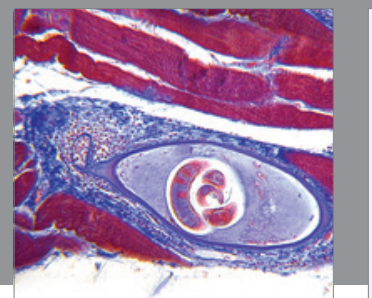

Gastroenterology

Research and Practice
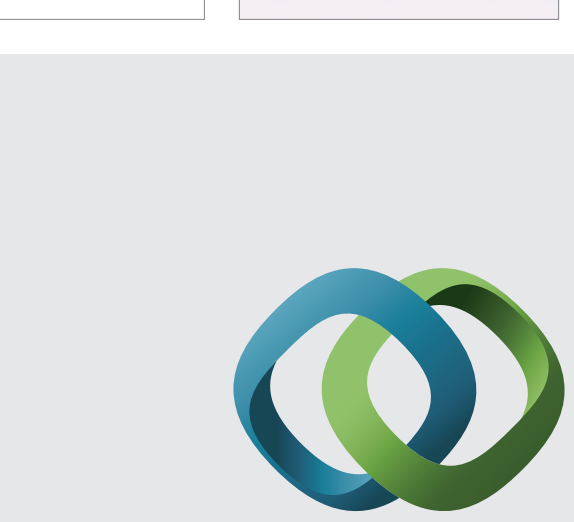

\section{Hindawi}

Submit your manuscripts at

http://www.hindawi.com
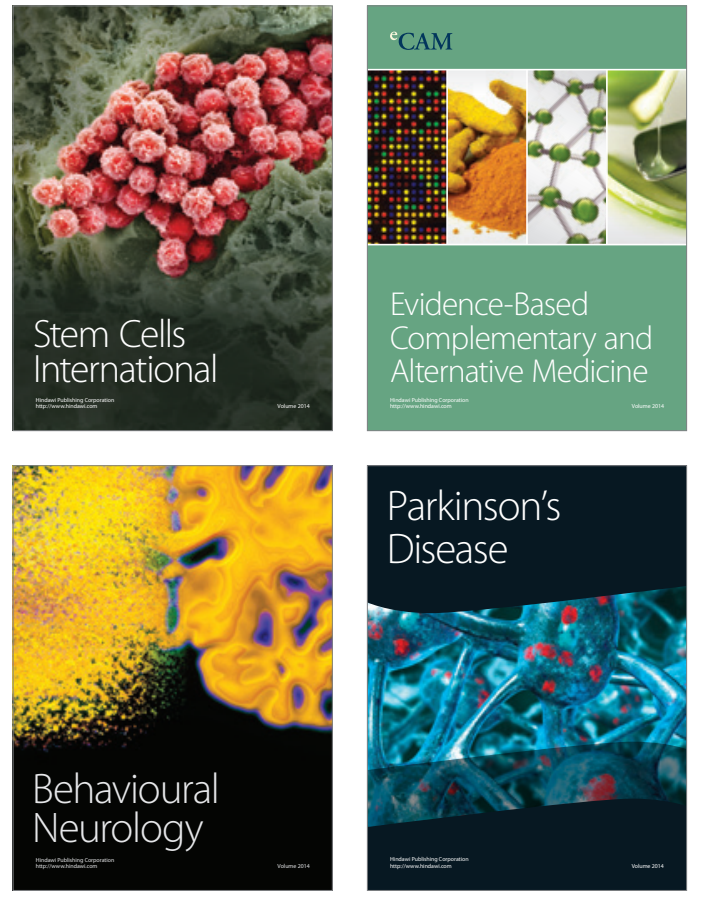
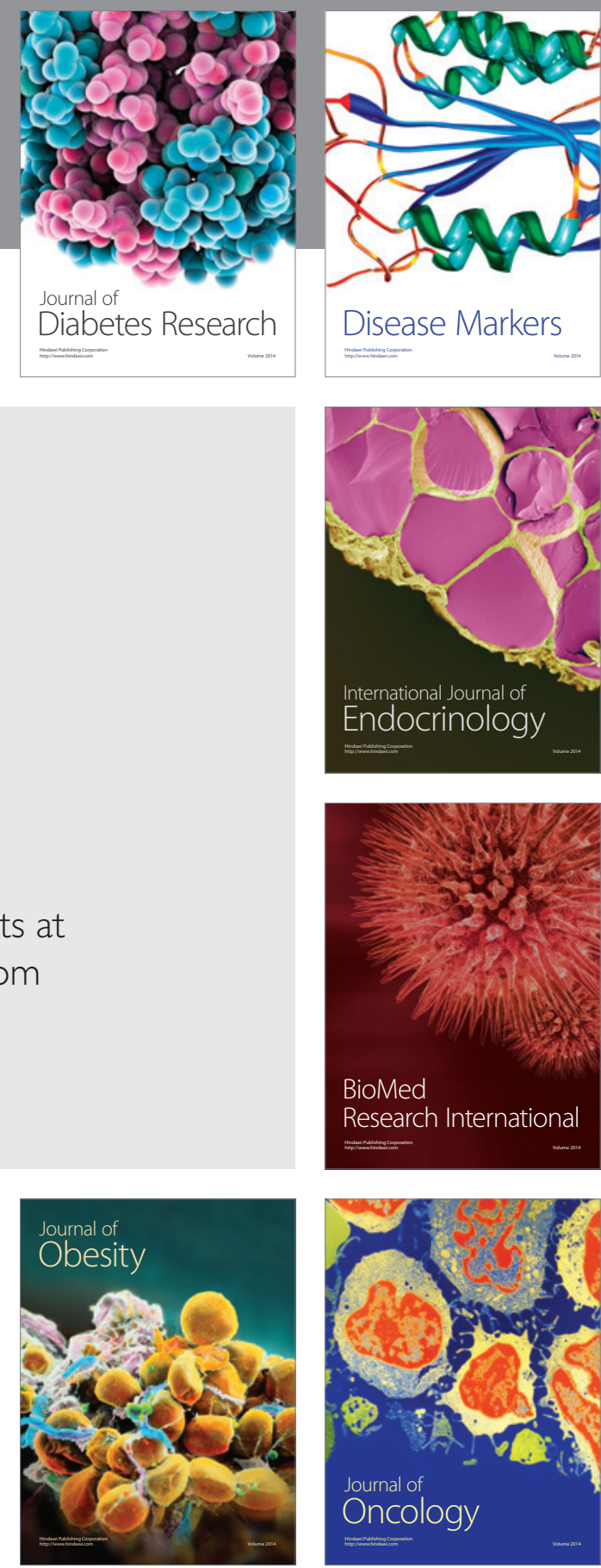

Disease Markers
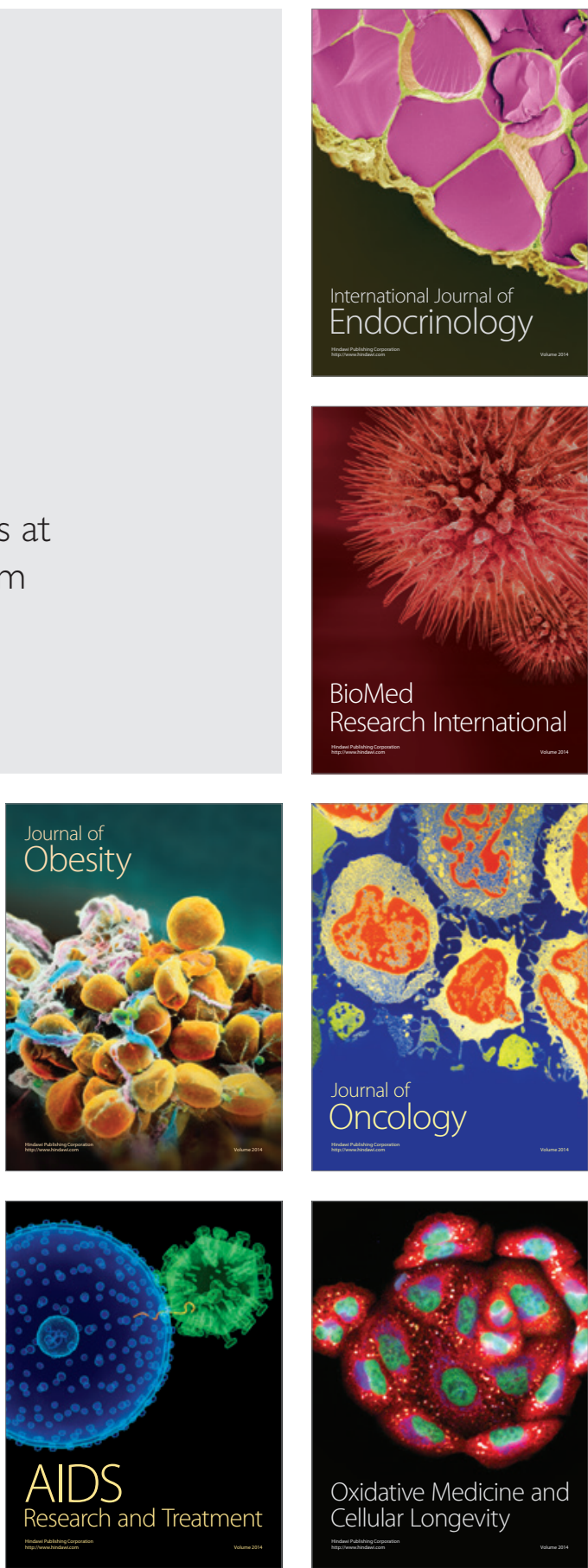